\title{
Politicas de salud. \\ Estrategia biopolitica para controlar \\ a los migrantes mexicanos en Estados Unidos
}

\author{
FERnANdo FARfán \\ IVONne Vizcarra \\ Norma GonZÁlez
}

Resumen. Este trabajo aborda las políticas de salud dirigidas a los migrantes que tienen como destino Estados Unidos, con el fin de controlar su acceso y permanencia en el país. El análisis se realiza desde el concepto de biopoder, propuesto por Michel Foucault, que se refiere a un poder cuyo objetivo es la sujeción de los cuerpos y el control de las poblaciones a través de técnicas y estrategias diversas. Así, las políticas públicas de salud constituyen el vehículo de control en torno a los migrantes mexicanos que van a Estados Unidos. Al respecto, se describen los dispositivos de acción y sus alcances, al tiempo que se verifica cómo se insertan estas políticas en los mecanismos globales para gobernar a la población migrante. Palabras clave: biopoder, biopolítica, migración, salud, gobierno.

\begin{abstract}
This paper analyses health policies aimed at migrants headed for the United States. The goal is to understand how these policies respond to strategies meant to control migrants access to and stay in the country. This is approached via Michel Foucault's notion of biopower: a power that aims to subject bodies and control of populations via a number of techniques and strategies. In this sense, public health policies constitute a vehicle to controls Mexican immigrants traveling to the United States. Their modes of action and their scope are addressed; moreover, these policies are examined in the context of global mechanisms used to govern the Mexican migrant population.
\end{abstract}

KeYwords: biopower, biopolitics, migration, health, government.

Fernando Farfán es profesor de la Facultad de Medicina de la Universidad Autónoma del Estado de México (UAEméx).

Ivonne Vizcarra es profesora Investigadora del Instituto de Ciencias Agropecuarias y Rurales de la UAEméx. Norma González es profesora Investigadora de la Facultad de Ciencias Políticas y Sociales de la uaeméx. 


\section{INTRODUCCIÓN}

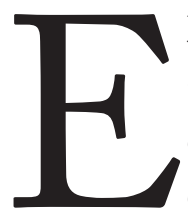
1 fenómeno migratorio entre México y Estados Unidos ha sido abordado desde múltiples aristas: a partir de las remesas, se analiza cómo los trabajadores mexicanos buscan trasladarse al vecino país del norte para obtener empleo y garantizar su supervivencia y la de sus familias. Heredia (2011), Delgado, Márquez y Rodríguez (2009), Levine (2006), entre otros, advierten sobre las condiciones laborales de los migrantes en Estados Unidos y la agudización de las políticas para controlar su incursión en territorio estadounidense, a partir de leyes más severas y mecanismos tecnológicos de vigilancia cada vez más sofisticados. En el mismo tenor, se han instrumentado políticas de salud, que igualmente pretenden controlar el flujo de migrantes, limitando su acceso a través de mecanismos más sutiles, como el Programa Vete Sano, Regresa Sano, en el que se verifican las condiciones de salud en los lugares de origen, tránsito y destino (ssa, 2002: 27). Una tendencia similar se observa en los requisitos, cada vez más drásticos, para la obtención de visas para inmigrantes que van a Estados Unidos.

No obstante, la medida biopolítica de mayor impacto sobre estas poblaciones consiste en la exclusión de los servicios de salud, aun cuando los trabajos que realizan los migrantes se lleven a cabo en empresas que cotizan por ley al gobierno estadounidense (Ponce, 2012). La contraparte se observa en la «omisión» de esas políticas restrictivas, cuando la necesidad de trabajadores se hace indispensable (Massey, 2009). Estas circunstancias, cotidianas en la relación entre México y Estados Unidos, constituyen el sustento de una biopolítica acomodaticia de ambos gobiernos, lo que Foucault describe como un poder que no sólo reprime, sino que también seduce, facilita, dificulta, amplía, limita y, por supuesto, prohíbe (Foucault, 2008: 148).

Dado que la salud es uno de los requisitos esenciales para un buen desempeño en materia laboral, el Estado desea controlarla a fin de garantizar el ejercicio pleno del poder, su legitimidad, además de practicar —si se

4 MigraCión Y DESARRollo, VOL. 10, NÚM. 19, SEGUNDO SEMESTRE DE 2012 
presenta la necesidad - el monopolio de la violencia para perpetuar el orden social. En este trabajo de reflexión teórica se parte de la siguiente hipótesis: en el siglo Xvir, el poder soberano correspondía a quien podía garantizar la vida de sus súbditos mediante el poder sobre la vida a través de la muerte; en la época contemporánea, el poder tiene la misma perspectiva de garantizar la vida, sólo que a través de una tecnología inversa, que consiste en hacer vivir o dejar morir (biopoder). Su ejercicio no lo efectúa un gobierno monárquico sino uno democrático que incide sobre las poblaciones para su control sobre el cuerpo y sobre la vida en general (Tejeda, 2011).

El objetivo de este artículo consiste en retomar la categoría explicativa de Michel Foucault sobre biopoder para adoptar una visión crítica sobre la migración mexicana actual y enriquecer la interpretación sobre el poder y las políticas de salud como mecanismos que ejerce el Estado. En el primer apartado se describen los conceptos de biopoder y biopolítica en sus distintas manifestaciones, desde sus inicios hasta finales del siglo XvII y principios del xvII; en el segundo apartado, se retoma la importancia del fenómeno migratorio y las medidas para contenerlo por parte de los gobiernos de México y Estados Unidos; en el tercero, se describen las características de salud de los migrantes mexicanos; en el cuarto apartado, se revisan las políticas de salud diseñadas para controlar a los migrantes en Estados Unidos y se problematiza el papel que adopta el Estado en la dominación de los cuerpos y la población migrante, por medio de políticas de salud excluyentes, tendencias que se unen con las políticas de mercado sobre las cuales debe debatirse. La justificación más importante de este trabajo se inscribe en la agenda nacional, donde el tema de los migrantes está pendiente como una de las grandes cuestiones a resolver.

\section{Estrategias DE BIOPODER}

El ejercicio del poder del Estado se manifiesta por las políticas públicas que pretenden controlar a los individuos y garantizar el orden social. Foucault esta- 
blece con claridad dos aspectos del poder que tienen que ver con la vida de los seres humanos: en primer lugar, un poder cuya finalidad consiste en «hacer morir o dejar vivir», facultad de la soberanía; en segundo lugar, un poder inverso al primero, cuya premisa consiste en «hacer vivir o dejar morir» y que se identifica como biopoder (Foucault, 2002: 165). Según nuestro autor, ese biopoder, cuyo objetivo consiste en administrar y controlar a las personas, se manifiesta en dos formas, una dirigida al control de las personas de forma individual, identificada como anatomopolítica, y otra dirigida a las poblaciones en su conjunto, la biopolítica. Para su ejercicio, ambas vertientes del biopoder se valen de minuciosas estrategias que varían según los objetivos que el poder pretenda. En Vigilar y castigar, Foucault explica cómo operan las técnicas anatomopolíticas: «A estos métodos que permiten el control minucioso de las operaciones del cuerpo, que garantizan la sujeción constante de sus fuerzas y le imponen una relación de docilidad-utilidad, es a lo que se puede llamar las disciplinas» (Foucault, 2004: 141). El sustento principal o brazo ejecutorio del control de las colectividades está constituido por las políticas gubernamentales de orden público que operan como disposiciones a través de leyes que se conforman como normas jurídicas de ejecución «obligatoria». En esa lógica, para entender cómo actúa el poder en la actualidad, se abordarán las políticas públicas de salud en torno a los migrantes mexicanos indocumentados, cuyo objetivo conlleva el control de estas poblaciones de no ciudadanos estadounidenses.

Si se parte del supuesto de que las políticas públicas atienden esencialmente las prioridades de la población más necesitada, se podrá ver en la práctica que, en no pocas ocasiones, dichas políticas no están encaminadas a tales fines, sino que su misión más importante consiste en proteger a los menos necesitados a partir de la aplicación de medidas sobre los más pobres, por ejemplo: «las intervenciones en los lugares insalubres, la aplicación de vacunas, los registros de las enfermedades, son medidas [...] que tienen por objeto el control de las clases sociales más necesitadas» (Foucault, 1999b: 384). Esta descripción corresponde a ciertas acciones del siglo xIX que, sin embargo, permanecen vigentes y son un claro ejemplo de estrategias biopolíticas de actualidad.

$6 \triangleleft$ MIGRACIÓN Y DESARROLLO, VOL. 10, NÚM. 19, SEGUNDO SEMESTRE DE 2012 
Desde las primeras descripciones del biopoder, particularmente en el ámbito biopolítico, Foucault hace notar cómo la salud se convierte en una estrategia central para el control de la población, cuando explica que «la gran medicina del siglo xix no se puede disociar de la organización [...], de una política de salud y de la consideración de las enfermedades en tanto que problema político y económico que se plantea a las colectividades, y que éstas deben intentar resolver a través de decisiones globales» (Foucault, 1999a: 328). Al considerar las enfermedades como problema político y económico, Foucault explicita que las respuestas deben ser globales, es decir, de orden biopolítico, en tanto que guardan relación directa con la vida de las personas.

El biopoder no sería posible si los progresos de la medicina y la biología no hubieran sentado las bases de su accionar a partir de la implementación de esquemas o estrategias de orden público para el cuidado de la vida, por ejemplo, el aislamiento de enfermos contagiosos cuyos padecimientos podían aniquilar poblaciones enteras, como la peste, la viruela o las patologías de carácter genético que podían trasmitirse de padres a hijos (fundamento esencial de la eugenesia) y la reordenación de la construcción de cementerios considerados como zonas de contaminación ambiental. Sin embargo, el instrumento más importante para el nacimiento de la biopolítica fue la cuantificación de nacimientos, enfermedades, muertes, etcétera. Con los datos estadísticos se ponen de manifiesto aspectos relativos a la sociedad en su conjunto (Foucault, 2001: 223). En el fondo, el interés de las políticas públicas no es proporcionar beneficios a la población en general, sino que se procede de forma selectiva. Históricamente, las estrategias biopolíticas han tendido a proteger a las clases más poderosas, aun cuando se pone en riesgo a otros grupos de «menor interés» para los gobiernos; ésa es la paradoja. Así, por ejemplo, el gobierno de Estados Unidos implementa políticas públicas (biopolíticas) que, ciertamente, beneficia a los ciudadanos estadounidenses, aunque perjudica a los inmigrantes. Tal es el caso de la reforma en materia de salud aprobada en marzo de 2010.

Aunado a la exclusión de la población no ciudadana estadounidense, la explotación de los migrantes es otro aspecto en su contra, ya que se les 
emplea en los puestos indeseables, peligrosos y menos remunerados (Delgado, Márquez y Rodríguez, 2009; Levine, 2006). El efecto más notorio de la estrategia biopolítica actual en contra de los migrantes en Estados Unidos consiste en la explotación laboral, la exclusión de los derechos humanos y el control para garantizar la productividad y la obediencia de los trabajadores inmigrantes. Sin embargo, este tratamiento conlleva algunas consecuencias, ya que los excluidos del derecho a la salud no podrán mantenerse sanos, menos aún con el tipo de trabajos que desempeñan. Por tanto, no estaría de más reconsiderar esta clase de estrategias pues, a la larga, cuando los trabajadores sean mayores y enfermen con más frecuencia, los resultados serán contrarios: menor será su capacidad para realizar los trabajos encomendados, habrá mayor ausencia por enfermedad y, consecuentemente, menor rendimiento.

Ante esa expectativa, resulta imperativo revisar las agendas bilaterales, con la finalidad de reestructurar las políticas a las que deben sujetarse los migrantes y así proveerles elementos necesarios para llevar una vida sana y digna. Empero, acorde con las tendencias políticas actuales, esta sugerencia más bien parece un sueño, ya que las medidas más severas para evitar el acceso de los migrantes a Estados Unidos están ocurriendo precisamente en esta época. E1 recrudecimiento de las estrategias biopolíticas ha llegado al grado de eliminar (parece el término más apropiado) a los migrantes cuando dejan de ser útiles por enfermedad, en lugar de proporcionarles los medios necesarios para su recuperación. La medida de eliminarlos se sustenta en un programa o, mejor dicho, en la biopolítica conocida como repatriación de connacionales enfermos, estrategia que consiste en enviar a México a los connacionales sin derecho a los servicios de salud, consecuente con el poco interés que las autoridades estadounidenses y mexicanas tienen para con los migrantes mexicanos, y que contrasta con la manifestación pública del propio gobierno estadounidense al expresar que «el importante número de mexicanos que labora en Estados Unidos constituye una contribución única al desarrollo económico y a la vida social y cultural en ese país» (ssa, 2010, 2008: 61). Aunque, en el fondo, ambas estrategias son congruentes con los objetivos del vecino país.

84 MIGRACIÓN Y DESARROLLO, VOL. 10, NÚM. 19, SEGUNDO SEMESTRE DE 2012 
De acuerdo con la afirmación de Heredia (2011): «Estados Unidos ha tratado de hacer frente al fenómeno migratorio por medio de una legislación interna que no discute (y menos aún negocia), a pesar de tratarse de una cuestión con múltiples aristas transnacionales». Caso contrario es el de México en torno al mismo tema, ya que «hasta los noventa, manejó su relación con el país vecino como "la política de la no política"» (Castillo, 2010; Urdanivia, 2011; Heredia, 2011). Durand explica que la política mexicana de la no política permanece vigente (Durand, 2011). Es tan manifiesto el desinterés por los migrantes en ambos países que no les importa generar estrategias públicas «a favor de éstos», a sabiendas de que las propuestas explícitas no se llevarán a la práctica. Es el caso de varios programas nacionales y binacionales de salud que, coincidentemente, tienden a delegar en los propios migrantes el cuidado de la salud, estrategia que la Organización Mundial de la Salud (oms) designa como autocuidado en la prevención de enfermedades (Ferrer-Lues, 2012).

Estos hechos evidencian cómo la salud es usada por el poder como instrumento de administración y control de las poblaciones más desprotegidas a favor de una ciudadanía que cuenta con mayores recursos. Otra estrategia frecuente consiste en proporcionar atención a «los sin derecho» cuando, por ejemplo, se trata de enfermedades infecto-contagiosas que pudieran, incluso, provocarles la muerte. Sin embargo, ese apoyo sanitario, que hace merecedoras del reconocimiento público a las autoridades e instituciones, tiene otros objetivos: evitan el contagio de una población mayor y, como resultado de estas acciones, mantienen bajo control a las personas enfermas y a sus familiares mediante sutiles mecanismos de vigilancia. El método de proporcionar apoyos a personas necesitadas es el ejercicio biopolítico cotidiano de los programas de salud para los migrantes indocumentados, como la Semana Binacional de Salud (sbs) y las Ventanillas de Salud (vs), cuyo propósito, orientado al autocuidado, corresponde a la estrategia biopolítica de elección. Esta estrategia se asemeja a lo que Foucault denomina el cuidado de sí: «El cuidado médico permanente es uno de los rasgos esenciales del cuidado de sí, hay que llegar a ser médico de sí mismo» (Foucault, 1999a: 456). 
Foucault sitúa el nacimiento del biopoder en el alba de la Revolución Industrial (finales del siglo xvII, principios del siglo xviII), etapa en la que se requería mano de obra obediente y bien preparada. A partir de este momento, se afinan las tecnologías de control de los cuerpos y se fortalecen las técnicas de disciplinamiento para moldear y construir los cuerpos de los trabajadores, de tal manera que cumplan cabalmente con las tareas a su cargo y se sujeten fielmente a las normas de las empresas; es el momento de las prácticas disciplinarias no sólo en las empresas laborales, sino en prácticamente todos los espacios públicos (reclusorios, escuelas, monasterios, fábricas).

En torno al rigor de los disciplinamientos, la formación o construcción de los soldados es un ejemplo claro:

\begin{abstract}
El soldado se ha convertido en algo que se fabrica; de una pasta informe, de un cuerpo inepto, se ha hecho la máquina que se necesitaba; se han corregido poco a poco las posturas; lentamente, una coacción calculada recorre cada parte del cuerpo, lo domina, pliega el conjunto, lo vuelve perpetuamente disponible, y se prolonga, en silencio, en el automatismo de los hábitos; en suma, se ha «expulsado al campesino» (Foucault, 2002: 139).
\end{abstract}

Mantener saludables los cuerpos de los «servidores» (trabajadores) y de sus familiares constituyó uno de los principios más importantes de la industria. El fordismo o Estado de bienestar keynesiano es, sin duda, una fiel manifestación de ello. Foucault (1988: 231) considera que, entre las estrategias más importantes para moldear y controlar a las poblaciones, la más efectiva es la subjetivación, ya que por ese medio se construye a los seres humanos bajo un esquema social de dominación y control a partir de distintos mecanismos. Mora (2010) coincide con este planteamiento de control a través del discurso sin violencia que, sin embargo, produce efectos en la voluntad de los dominados, donde la construcción social de los individuos es exigir que se rindan ante el poder, obedezcan y asuman dicha relación como 
natural. La subjetivación a la que Foucault hace referencia se sustenta en discursos de «verdad», aduciendo que el poder no sólo es represivo, ya que de este modo difícilmente se podría sostener, sino que también induce placer, produce cosas, no se limita a decir no (Foucault, 2008: 148).

En tal sentido, el interés de este estudio busca esclarecer los mecanismos utilizados por los gobiernos mexicano y estadounidense para ejercer el biopoder institucional (biopolítica) sobre los cuerpos de los migrantes mexicanos en Estados Unidos, partiendo de la idea de que el poder no es únicamente el efecto del ejercicio de una autoridad, sino un haz de discursos y prácticas que convergen hacia la dominación y control de los cuerpos (Foucault, 2002: 114). Para el caso de los migrantes, lo que está en juego es la propia vida. El alcance o, mejor dicho, la orientación de las estrategias gubernamentales implementadas para hacerlos vivir o dejarlos morir usa como engranaje este esquema del aspecto de la salud a través de las propuestas políticas sobre la misma, pues una forma de ejercer el poder es mediante la política, que tiene como fin último dominar y controlar la vida de los sujetos sociales a través del cuerpo y del pensamiento, con técnicas sutiles o coercitivas (Gil, 2009).

Semejante a lo que acontecía con el poder soberano, las leyes actuales otorgan al poder el legítimo derecho para preservar la vida y promoverla a través de su administración, y establecer una conformación de seguimiento y vigilancia durante todo el proceso de la vida. La política se convierte en biopolítica cuando el binomio vida-muerte declina a favor de la primera, generando una dinámica en la que están inmersos los migrantes, gobernados y administrados políticamente.

Giorgio Agamben abona al tema de la biopolítica a partir de su trabajo sobre la inclusión de la vida biológica en los mecanismos del Estado, considerando la esencia misma de todas las formas de poder en Occidente. La especie y el individuo, en cuanto cuerpos vivientes, se convierten en objetos de las estrategias del poder político que instrumenta políticas de atención o exclusión propias de los gobiernos actuales (Agamben, 2010). 
De este modo, uno de los centros de atención de la política contemporánea se ha situado en la biopolítica. El control del Estado sobre el cuerpo de los individuos se manifiesta, por ejemplo, en la prohibición del aborto, la tolerancia sexual, la eutanasia, nuevas formas de procreación, así como en directrices específicas sobre quiénes tienen acceso a la salud; Esto último puede plantearse para el control de los migrantes mediante procesos biológicos.

Así, los individuos deberán asumir que su vida es controlada por el Estado, el cual otorga los servicios de salud y, en un momento dado, puede retirarlos o restringirlos arbitrariamente por el imperio de la ley. Agamben (2010) advierte que este control se da a partir de un Estado de excepción y establece que en el orden legal los individuos pueden estar dentro o fuera de la ley: eso es lo que distingue su acceso a la vida. En el caso de los migrantes, el acceso a la salud está vinculado con los documentos que acrediten su estancia legal en Estados Unidos. Todos aquellos migrantes que no cuenten con tal requisito son excluidos «legalmente» de la mayoría de los beneficios a que un ser humano tiene derecho, incluidos, entre éstos, el acceso a los servicios de salud.

De esta forma se constata que la biopolítica es un mecanismo adoptado por el Estado para garantizar el control de los cuerpos, no como súbditos - característica del poder soberano- sino como ciudadanos sin derechos, condición en la que se encuentran más de $50 \%$ de los migrantes mexicanos en Estados Unidos (emif, 2011; uc y ssa, 2007: 21), debido a que carecen de la ciudadanía estadounidense a pesar de cubrir puntualmente las cuotas o impuestos establecidos por el gobierno a todos los trabajadores.

\section{Fenómeno MigRatorio}

Históricamente, México ha sido una fuente de trabajadores que buscan mejores condiciones de vida en Estados Unidos. Este fenómeno ha sido estudiado por múltiples investigadores (Durand, Massey, Delgado, Santibáñez, Tuirán, entre otros), quienes coinciden en que los movimientos migratorios

124 MIGRACIÓN Y DESARROLLO, VOL. 10, NÚM. 19, SEGUNDO SEMESTRE DE 2012 
de mexicanos a Estados Unidos están relacionados, en buena medida, con las disparidades económicas y salariales entre México y Estados Unidos, amén de las necesidades de mano de obra por parte de los empresarios estadounidenses, lo que origina, como menciona Massey (2009), vaivenes en torno a la vigilancia y el acceso al territorio estadounidense, manifiesto por la facilidad o dificultad que representa para los migrantes cruzar la línea fronteriza. Así, cuando la mano de obra se hace más necesaria, el número de migrantes documentados o no- se incrementa; el caso contrario ocurre cuando los requerimientos de trabajadores son menores. Otro aspecto de coincidencia entre los que investigan sobre este fenómeno tiene que ver con la carencia de derechos de los migrantes en territorio estadounidense, cuestión que a la fecha es motivo de discusión entre los gobernantes de ambos países.

\section{CUADRO I}

Población nacida en México, residente en Estados Unidos según sexo 2000-2008.

\begin{tabular}{cccc}
\hline AÑo & TOTAL & HOMBRES & MUJERES \\
\hline 2008 & $11,657,266$ & $6,497,339$ & $5,159,927$ \\
2007 & $11,895,657$ & $6,667,862$ & $5,227,813$ \\
2006 & $11,695,228$ & $6,536,156$ & $5,159,072$ \\
2005 & $11,169,112$ & $6,211,409$ & $4,957,703$ \\
2004 & $10,404,919$ & $5,738,773$ & $4,666,146$ \\
2003 & $10,241,301$ & $5,509,483$ & $4,508,004$ \\
2002 & $10,017,487$ & $5,509,483$ & $4,508,004$ \\
2001 & $9,403,069$ & $5,203,968$ & $4,199,101$ \\
2000 & $9,023,756$ & $4,977,486$ & $4,046,270$ \\
\hline
\end{tabular}

Fuente: estimaciones oficiales del INEGI con base en U.s. Census Bureau, Encuesta de la Comunidad Americana (ACS), 2008.

Los vaivenes del fenómeno migratorio a los que hace referencia Massey han estado presentes durante toda la historia de la migración mexicana hacia Estados Unidos. No obstante, las crisis económicas de la presente dé- 
cada a nivel mundial han repercutido de forma notoria en el tránsito y estadía de los migrantes en el país vecino, ya que, a partir de esos hechos, la política de Estados Unidos adoptó acciones más severas, con la finalidad de evitar que más migrantes indocumentados lograran colocarse como trabajadores. A pesar de ello, el número de migrantes mexicanos tuvo un crecimiento constante desde 2000 hasta 2007, cuando alcanzó la cifra máxima de la década, logrando casi los doce millones (véase cuadro 1), lo cual ciertamente resulta paradójico, ya que para ese tiempo las restricciones para ingresar al territorio estadounidense eran más severas, como consecuencia de los atentados del 11 de septiembre de 2001.

Otro aspecto que ha sido una constante para los migrantes mexicanos es el maltrato y el tipo de empleos. Levine (2006: 97) menciona al respecto: «No es extraño que inmigrantes recientes ocupen los puestos de trabajo menos deseables con los salarios más bajos de Estados Unidos que, sin embargo, representan mucho más de lo que podrían ganar en sus países de origen». Delgado, Márquez y Rodríguez (2009) coinciden con Levine aduciendo además que «los migrantes mexicanos ocupados carecen de una amplia gama de servicios sociales: la gran mayoría no tiene acceso al esquema de seguridad social ni a los programas de asistencia pública». Cabe destacar que las opiniones de los investigadores en este sentido son ratificadas por Aguilar, comisionada del Instituto de los Mexicanos en el Exterior (IME), quien expresa:

De ser individuos con derechos y prerrogativas, nos convertimos en infractores de la ley, en perseguidos, en objeto de acciones de crímenes y odio; en trabajadores que están obligados a pagar impuestos allá, pero que no son reconocidos para tener acceso a los servicios básicos de salud; no tenemos las mismas condiciones de acceso a todos los niveles de educación escolar; a salarios equitativos. Qué paradójico, podemos comprar autos, pero no podemos tener licencias de conducir, podemos tener un número de identificación tributaria para pagar impuestos, pero no po-

14 MigRACión y DESARROLLO, VOL. 10, NÚM. 19, SEGUNDO SEMESTRE DE 2012 
demos tener un número de seguro social que nos dé acceso a beneficios plenos (Aguilar, 2010).

Durand va un poco más allá y puntualiza que la estrategia mexicana conocida como «la política de la no política», que consiste en hacerse omiso a este tipo de problemas, es una política de lavarse las manos. Al fin y al cabo, resulta de «interés nacional» que los mexicanos busquen oportunidades en el país vecino, lo que ha sido calificado como una «válvula de escape» para los problemas económicos, políticos y sociales del país (Durand, 2011). La respuesta de Estados Unidos es semejante, ya que las leyes imperantes son propias y, como menciona Massey (2009): «la historia confirma que el proceso migratorio entre México y Estados Unidos ha sido forjado por las políticas unilaterales de Estados Unidos».

Aunado a esta añeja problemática, los actos terroristas del 11 de septiembre de 2001 han dejado prever, para el gobierno estadounidense, que es indispensable una ley al respecto, ya que, al margen del gobierno federal, cada vez son más los estados de Estados Unidos que están generando sus propias leyes, en las que parece que la «norma» consiste en criminalizar a los migrantes mexicanos. Es tal el descontento de algunos ciudadanos estadounidenses, que «a lo largo de la presente década los estados han buscado hacer su propia política migratoria. Sólo en 2010, las legislaturas estatales consideraron más de 1,500 iniciativas relacionadas con el tema migratorio» (De los Ríos, 2011). Sin embargo, todos esos mecanismos habían sido insuficientes para contener la avalancha de mexicanos indocumentados en busca del «sueño americano» hasta antes de 2008, año en el que la emigración decreció notablemente, aunque continúe dándose (Massey, 2009).

Es claro que la población migrante más afectada es la de los indocumentados; sin embargo, son éstos los que en la última década han crecido en mayor proporción. En la gráfica 1 se puede observar que, a excepción de 2004, de manera casi sistemática, se incrementó la emigración de indocumentados mexicanos de 2002 a 2007, pasando de 330 mil a 683 mil. Caso contrario sucedió con 
los migrantes legales, que de 397 mil en 2002 descendieron a 148 mil en 2006. Estos datos muestran que de una u otra forma (legal o indocumentada) los migrantes continúan trasladándose hacia el vecino país y que la inversión en la forma de incursionar a territorio estadounidense se da como consecuencia de la reducción del número de permisos para introducirse legalmente; asimismo, se observa que a partir de 2008 comienza a darse un decremento notorio de la emigración indocumentada, siendo en en el año 2010 mínimo el número de personas que intentan cruzar la frontera sin documentos.

\section{GRÁFICA I}

Flujos migratorios laborales México-Estados Unidos, total e indocumentados, 2002-2010.

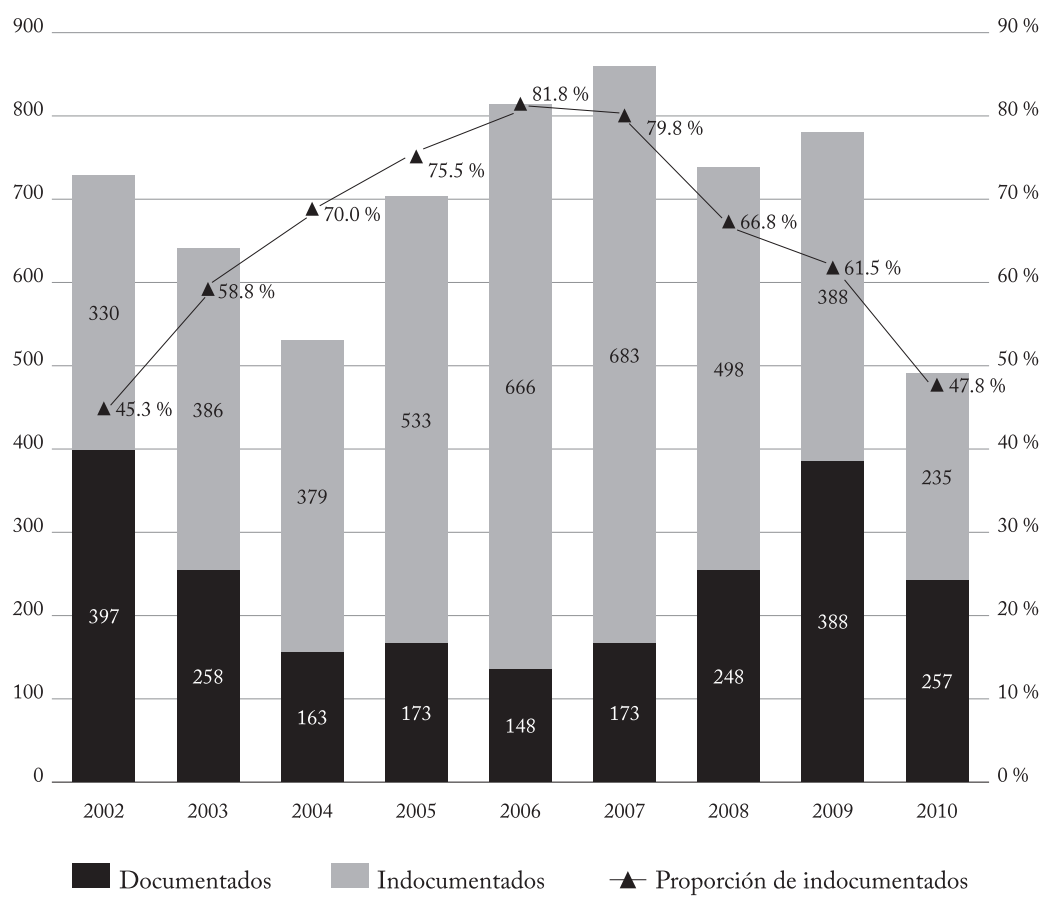

Fuente: estimaciones de Conapo con base en el Conapo, INM, SER, STPS y

el Colef. Encuesta sobre Migración en la Frontera Norte de México (emif norte), 2002-2010. 
Otro aspecto que no puede soslayarse es el envío de remesas de estos trabajadores a México. En 2007, el Banco Interamericano de Desarrollo (BID) estimó en 63 mil millones de dólares el flujo de divisas hacia América Latina, de las cuales un tercio correspondió a México (BID, 2010). Ciertamente, estas remesas permiten sostener un gran número de familias mexicanas y la economía de varias regiones. Entonces se deduce que es debido a tal motivo que las políticas sean permisivas, tolerantes, y que ninguno de los gobiernos involucrados ataque el problema de fondo, pues ello significaría el reconocimiento y la aceptación legal que conllevaría a cuestionar la forma en que son tratados los migrantes en Estados Unidos. Esto no sucede por la asimetría de poder entre México y el vecino país del norte.

\section{SAlud De Los Migrantes}

La migración indocumentada hacia Estados Unidos implica, de facto, la exclusión por el solo hecho de no ser ciudadano de aquel país. Entre otras cosas, se excluye a los indocumentados del derecho a la salud, indispensable para estos trabajadores, ya que se trata de su principal activo. «Para quien tiene como único medio de subsistencia su fuerza de trabajo físico e intelectual, su estado de salud es la única reserva, su único capital» (Miglionico 2006: 15). El buen estado de salud de los migrantes es requisito indispensable para conseguir empleo y garantizar la posibilidad de ser contratado e incorporado en la economía estadounidense bajo sus reglas y condiciones, generalmente desfavorables para los trabajadores migratorios.

$\mathrm{Al}$ respecto, cabe mencionar que los migrantes mexicanos que salen de sus poblaciones de origen a laborar en Estados Unidos son, en general, personas jóvenes y saludables (Uc, 2007: 13), condición que concuerda con los padecimientos que mayormente afectan a las poblaciones de emigrantes en México (véase cuadro 2), que en su mayoría son de tipo «benigno», de tratamiento generalmente efectivo y de corta duración, a excepción de la 
hipertensión arterial y la diabetes mellitus, que ocupan los lugares siete y nueve, respectivamente, enfermedades que no son benignas pero que pueden tratarse de forma efectiva cuando se detectan a tiempo y no originan complicaciones en la mayoría de los casos.

\section{CUADRO 2}

Diez primeras causa de morbilidad en México, 2008

\begin{tabular}{cc}
\hline ORDEN DE & CAUSA DE MORBILIDAD \\
FRECUENCIA & Infecciones respiratorias agudas \\
\hline 1 & Infecciones intestinales por otros organismos y las mal definidas \\
3 & Infecciones de vías urinarias \\
4 & Úlceras, gastritis y duodenitis \\
5 & Otitis media \\
6 & Amibiasis intestinal \\
7 & Hipertensión arterial \\
8 & Gingivitis y enfermedad periodontal \\
9 & Diabetes no insulinodependiente (tipo 2) \\
10 & Otras helmintiasis \\
\hline
\end{tabular}

Fuente: ssa, 2008.

Contrario a lo anterior, se puede observar en el cuadro 3 que las causas más comunes de enfermedad entre los migrantes latinos que viven en Estados Unidos son padecimientos que en su mayoría requieren de largos tratamientos o cirugías, así como hospitalizaciones frecuentes. Estos aspectos patológicos contrastantes entre las poblaciones de origen de los migrantes y Estados Unidos probablemente sean consecuencia del cambio de estilos de vida, de los empleos que desempeñan y, por supuesto, de un cierto descuido en la prevención y atención de enfermedades en el momento oportuno debido a la inaccesibilidad a los servicios de salud para estos migrantes, rubro en el que más de 55\% no cuenta con esta prestación (EMIF, 2011; uc y sSA, 2008: 21). 
CUADRO 3

Diez primeras causa de morbilidad en Estados Unidos

\begin{tabular}{cc}
\hline ORDEN DE FRECUENCIA & CAUSA DE MORBILIDAD \\
\hline 1 & Diabetes \\
2 & Obesidad \\
3 & Hipertensión \\
4 & Tuberculosis \\
5 & Estrés-depresión \\
6 & Cáncer cérvico uterino \\
7 & Cáncer de mama \\
8 & Enfermedades actividad laboral \\
9 & Cáncer de próstata \\
10 & Sida \\
\hline
\end{tabular}

Fuente: ssA, 2008.

Ante tan elevados índices de desprotección en salud entre los migrantes mexicanos en Estados Unidos, es indispensable que los gobiernos, sobre todo el mexicano, promuevan alternativas que permitan a estas personas asistir, al menos, a la consulta médica privada sin el riesgo de que por este medio sean detectados y deportados, ya que ese es uno de los mayores temores de los migrantes indocumentados. Esto facilitaría, además, la asistencia oportuna en los Centros de Salud para atender con prontitud problemas que pueden ser de gravedad. Por lo pronto, ante la carencia de apoyos para su atención, las alternativas más comunes son el uso de remedios caseros, la automedicación, las llamadas telefónicas a familiares para pedir consejo, el traslado a las ciudades fronterizas más cercanas para atenderse en México, entre otras (Nigenda, 2009).

Las condiciones de vida y salud a las que están sometidos los migrantes mexicanos indocumentados en Estados Unidos —ocasionadas por un poder velado o, mejor dicho, amparado en las leyes de su país, en las que el ejercicio del biopoder está presente (limitando o excluyendo a los migrantes de los servicios de salud, además de los empleos) — obligan, como se 
mencionó anteriormente, a una actuación decidida del gobierno mexicano que tienda a subsanar, en lo posible, las carencias de estos coterráneos para mejorar sus condiciones de vida. Para tal efecto, el primer paso sería la protección de estos trabajadores al interior de las empresas en las que laboran; el segundo, y más importante, sería la concurrencia de acuerdos bilaterales entre los gobiernos mexicano y estadounidense, con la finalidad de implementar las estrategias necesarias para cubrir las demandas más urgentes en materia de salud. Las respuestas de ambos gobiernos, como se ha comentado, no dan pie a cambios sustantivos. No obstante la disposición del gobierno mexicano, como se apreció en algún momento durante el periodo presidencial de Vicente Fox (2000-2006), los acuerdos binacionales no culminaron, quedando sólo en buenos propósitos. La Declaración Conjunta sobre Salud del Migrante entre la Secretaría de Salud de los Estados Unidos Mexicanos y el Departamento de Salud y Servicios Humanos de Estados Unidos (DC) es el ejemplo más emblemático de los acuerdos y desacuerdos entre ambos gobiernos, ya que, a pesar de haberse firmado por las máximas autoridades sanitarias de ambos países, los resultados fueron mínimos.

Algunos ciudadanos de Estados Unidos consideran como algo normal que los migrantes no tengan derecho a los servicios de salud; tienen la idea de que éstos representan una sangría para la economía de su país, lo cual ha sido desmentido puntualmente (Heredia, 2011; Pérez, 2008) al demostrarse que las aportaciones que hacen los migrantes indocumentados son mayores que los beneficios que reciben. El imperio del biopoder, amparado en las políticas gubernamentales, difícilmente cambiará de estrategia en el trato para con los migrantes mexicanos, si bajo este esquema consiguen la realización de sus propósitos.

Ante este tipo de experiencias y los antecedentes del programa Bracero, Durand propone que para nuevos acuerdos bilaterales se consideren, como responsabilidad del país que acoge, salarios similares a los que perciben los ciudadanos residentes de los lugares donde lleguen a laborar los migrantes, seguro médico y seguro de desempleo, entre otras garantías de interés. Particular- 
mente, sobre el programa Bracero, indica que la corrupción de las autoridades mexicanas obstaculizó algunos acuerdos con Estados Unidos (Durand, 2007).

\section{Políticas de SALud y Biopoder}

Después de casi ciento cincuenta años de relación bilateral entre México y Estados Unidos, tiempo transcurrido igualmente para el fenómeno migratorio entre ambos países, no se han logrado los acuerdos que produzcan un trato digno a los migrantes mexicanos que, por necesidad y bajo la anuencia de ambos gobiernos, cruzan la línea fronteriza para laborar en territorio estadounidense. Sin embargo, la conveniencia binacional —entendida por el lado mexicano como «la política de la no política» y por el lado estadounidense como la imposición de sus puntos de vista bajo la estrategia de no discutir ni negociar sus políticas migratorias - ha tenido resultados fatales, ya que hasta 1961 se dieron los primeros pasos para la construcción de una relación formal a través de las Reuniones Interparlamentarias entre los representantes de los poderes legislativos de ambas naciones. En 1981 se constituyó la Comisión Binacional México-Estados Unidos, originalmente «sólo» con las Secretarías de Relaciones Exteriores, Comercio y Hacienda; en 1989 se incorporaron las comisiones de todas las secretarías de Estado, entre ellas la de Salud (Becerra, 2004). Corto es realmente el tiempo de interacción formal entre México y Estados Unidos - aproximadamente veinte años-, lo que podría de algún modo justificar la falta de acuerdos en el rubro que nos atañe; no obstante, lo más alarmante es que, pese a la claridad y urgencia del problema, las propuestas para afrontarlo sean tan limitadas o se generen para otros fines.

Ciertamente, se podrá argumentar que existen varios programas de salud orientados a la prevención y la protección de la salud de los migrantes mexicanos en Estados Unidos, empero, antes de abocarnos a ello, vale aclarar que a partir de marzo de 2010 se elimina de tajo toda posibilidad de que los no ciudadanos accedan a los servicios de salud, con la promulgación de la 
nueva ley de salud estadounidense, en la que, por una parte, se exige la inclusión de todos los ciudadanos a la seguridad en salud y, por la otra, se impide que los migrantes indocumentados y los legales con menos de cinco años de estadía en el país puedan adquirirla por medios propios (Bossert, 2010; Observatorio de la migración, 2010).

Este mecanismo de segregación de la población indocumentada es ejemplo manifiesto de las estrategias biopolíticas, que tienen como fin último el control y el aprovechamiento de los migrantes, recurso que no es nuevo pero sí efectivo para los intereses estadounidenses; es una práctica que Agamben (2012) describe como una «vida sin calificación, la vida en su estado desnudo, salvaje, podríamos decir, especialmente desde la perspectiva de la vida política y social». Aun así, esta vida puede cambiarse, ya que la libertad de los migrantes, requisito indispensable para la práctica del biopoder, no está totalmente coartada (Foucault,1988: 238), aunque la alta vulnerabilidad a la que están expuestos limite su accionar y reduzca su posibilidad de respuesta a la «esperanza» de que las autoridades mexicanas hagan algo por ellos. También les queda, en su defecto, la alternativa de retornar a territorio mexicano, como lo están haciendo varios miles desde 2008.

Ante la emisión de esta ley, cuya aplicación integral culmina en 2019, se reduce más la posibilidad del ejercicio de los programas de salud dirigidos a los migrantes mexicanos que viven en Estados Unidos; programas que, para ser honestos, han servido más como escaparate político que como acciones en beneficio de los migrantes, ya que, en estricto rigor, sólo dos de ellos tienen efecto directo sobre esta comunidad (s bs y vs). Por otra parte, el programa de Repatriación de Enfermos no es de utilidad en Estados Unidos.

Los programas de salud publicados, aunque no propiamente ejercidos, son ocho: Declaración Conjunta (DC) de 2000; Iniciativa de Salud MéxicoCalifornia (ismecal) de 2001; Semana Binacional de Salud (sbs) de 2001; Vete Sano, Regresa Sano (vsrs) de 2002; Ventanilla de Salud (vs) de 2002; Repatriación de Connacionales Enfermos (RCE) de 2002, y Salud del Migrante (sm) de 2008. 
Llama la atención el hecho de que sean tantos los programas dirigidos a la población migrante indocumentada, además de que hayan sido publicados en un periodo tan corto. Esto se puede explicar si se parte del hecho de que el primer acuerdo binacional de este tipo fue avalado por la firma de los responsables de la salud tanto de México como de Estados Unidos. En dicho documento se establece una serie de compromisos para los migrantes mexicanos en el vecino país. Se confiere especial interés a éste que la ssa difunde en los términos siguientes:

E1 22 de septiembre de 2000, el Secretario de Salud de México y la Secretaría de Salud y Servicios Humanos de Estados Unidos suscribieron la Declaración Conjunta sobre Salud del Migrante en la que ambos países manifestaron su interés en desarrollar actividades de cooperación para atender las necesidades de salud que enfrentan los migrantes y sus familias, y se comprometieron a fortalecer la colaboración binacional sobre la salud del migrante.

Este documento constituye un parteaguas en la medida en que se reconoce por primera vez la importancia social y económica de los trabajadores mexicanos en Estados Unidos. Cito: «el importante número de mexicanos que labora en Estados Unidos constituye una contribución única al desarrollo económico y a la vida social y cultural en ese país».

De esta manera, el documento reconoce de manera explícita que el proceso migratorio es de naturaleza binacional y, por tal motivo, responsabilidad de ambos países (ssa, 2010; ssa, 2008: $57-63)$.

Sin embargo, aún cuando en la práctica no hubo sustento de dicho programa para explorar los campos de la posible acción bilateral, es importante recalcar que en el documento se reconoce explícitamente que el proceso migratorio es de naturaleza binacional y, por tal motivo, responsabilidad 
de ambos países, siendo enfático el tema de la salud de quienes migran hacia Estados Unidos. Se trata de un documento amplio, en el que se puede observar con claridad un control de estas personas, incluso desde antes de partir, en sus lugares habituales, en los que son identificados y seguidos durante su trayecto y hasta su destino; es decir, se pone un cuidado extremo que proporcione elementos suficientes para una "precisa selección» de quiénes deben cruzar la frontera y quiénes no. A pesar de la no ejecución del mismo, sus efectos fueron trascendentes para la creación de otros programas sobre la salud o cuidado de los migrantes, como ISMECAL, SBS, vSRS, vs, RCE y SM. En todos ellos se detectan elementos constitutivos de la DC, lo cual pone de manifiesto que el interés estadounidense está presente, aún cuando se pregone que VSRS, vS, RCE y SM son de origen mexicano. Esta condición da sustento a los casi nulos resultados de dichos programas en la práctica, consecuente con la falta de acuerdos entre los gobiernos en cuestión.

En el discurso de cada programa se conciben propuestas verdaderamente interesantes. Así, por ejemplo, vsrs ofrece un blindaje en salud para los migrantes en los lugares de origen, tránsito y destino; vs ofrece información sobre los centros de salud u hospitales a los que pueden acudir los migrantes, donde los costos de atención son menores y se confía en que no se comunicará a ninguna persona sobre su estatus migratorio. sBs es, al parecer, el programa que mayor difusión ha tenido, ya que desde el principio estaba focalizado en el estado de California y algunas ciudades fronterizas mexicanas; a la fecha se conoce como Iniciativa de Salud de las Américas, ya que su crecimiento ha sido tal que abarca toda la nación de Estados Unidos, México, Canadá y otros países de Centroamérica.

El formato de las acciones de sBs es de dos tipos: una netamente política, conocida como Foro de Políticas Públicas, y otra identificada como Ferias de Salud. En el primer tipo de acción se tratan temas diversos, con una tendencia hacia la atención de la salud de los migrantes, especialmente en Estados Unidos. Cabe mencionar que desde la primera sвs (2001, en Fresno, California) se propuso la creación de seguros binacionales de salud, 
propuesta que, a la fecha, no se ha logrado, lo que permite inferir lo siguiente: pese a que altas personalidades del ámbito político y gubernamental de los países inmersos asisten a este foro, éstas no cuentan con la autoridad para proponer y decidir qué hacer en casos tan importantes como el que se menciona. En las Ferias de la Salud —el segundo tipo de acción — se ofrece a los migrantes diversas acciones de salud, como exámenes para la detección de enfermedades, aplicación de vacunas, revisiones dentales, promoción para la salud, dotación de preservativos y otros. Todas éstas son acciones gratuitas; no obstante, existe el riesgo de que la información sea usada para conocer aspectos «inconvenientes» para los migrantes, por ejemplo, conocer si una persona goza de buena salud, si está en riesgo de padecer alguna enfermedad o algún padecimiento de interés para la salud pública, como tuberculosis, VIH/sIDA, etcétera. En otro sentido, las acciones de promoción para la salud pueden utilizarse igualmente como estrategias biopolíticas para controlar a las personas de forma tal que se les haga responsables de su enfermedad o de su salud. Todas estas acciones pueden considerarse de tipo biopolítico porque están sustentadas en estatutos legales.

Es evidente que mientras los migrantes sean jóvenes y sanos tendrán alguna oportunidad para acceder legalmente, incluso como indocumentados, a territorio estadounidense por la conveniencia que ello representa: la «inversión» binacional es mínima y la ganancia es alta. Sin embargo, cuando esta población va envejeciendo, las enfermedades se hacen presentes, pues a mayor edad más probabilidades de enfermarse, $y$, desgraciadamente, las patologías más graves ocurren con más frecuencia en las etapas tardías de la vida. Estas condiciones (edad avanzada y enfermedad) de los migrantes son, al parecer, el punto más álgido para el concurso de acuerdos entre los gobiernos mexicano y estadounidense. Se ha mencionado que, de manera general, los migrantes reditúan beneficios para ambos países. Mas no se ha considerado que hasta antes de llegar a Estados Unidos, estos migrantes han requerido de cuidados familiares y apoyos gubernamentales para un crecimiento y desarrollo adecuado. Estados Unidos los recibe listos para el trabajo; en con- 
secuencia, parecería lógico que el país para el que laboran y al que entregan su salud les proporcione los apoyos necesarios para lograr una vida digna; pero los apoyos son mínimos y, como se ha mencionado, México, por medio del programa RCE, ha tomado la decisión de hacerse cargo de ellos cuando están enfermos y ya no son de utilidad para las empresas en las que laboran, en pocas palabras, cuando han dejado de ser rentables para Estados Unidos.

Sería interesante investigar más a fondo el funcionamiento de este programa, ya que no se conoce mucho sobre el mismo y las repercusiones para el país y los migrantes podrían ser fatales. Este programa ejemplifica de modo fehaciente la práctica biopolítica; se crean las condiciones de conveniencia del poder: por un lado, las leyes que excluyen a los migrantes y, por otro, la puesta en marcha de un programa que deslinda a ese poder de la responsabilidad para atender a los migrantes. Esta estrategia biopolítica es coincidente con la propuesta foucaultiana en el sentido de que el biopoder administra la vida y ejerce sobre ésta controles y regulaciones de gran precisión (Foucault, 2002: 165).

La contraparte de las anteriores propuestas corresponde a la Reforma de Salud de Estados Unidos aprobada en marzo de 2010. Esta política unilateral — referida anteriormente - tiene la característica de avanzar paso a paso para quedar finiquitada en 2019. A través de esta estrategia se pretende incorporar al grueso de la ciudadanía estadounidense en los seguros de salud gubernamentales, dejando en el desamparo a todos los inmigrantes indocumentados e, incluso, a los documentados con menos de cinco años de residencia en Estados Unidos; además, no autoriza que estos dos grupos de migrantes adquieran seguros médicos privados. Esta reforma ha llegado a su segundo año de vigencia y desconocemos las condiciones en que los migrantes sin derechos subsisten a esta ley, pero atendiendo a lo que más de un investigador indica respecto a que Estados Unidos no discute sus pronunciamientos, lo esperado es que cuando se cumpla el plazo de «gracia» (2019) las deportaciones serán masivas. Volvemos, entonces, a las estrategias biopolíticas: más que hacer vivir a los migrantes, se los está administrando con la 
intención de sacarles el mayor beneficio y extraditarlos, llegado el momento. Todavía quedan siete años y en ese tiempo seguramente ocurrirán cosas, esperamos que no sean en demérito de los migrantes.

Finalmente, no se comprende por qué siendo tantas las personas afectadas por esta ley - 7.5 millones para 2010 (CONAPo, 2011: 251)—, ninguno de los dos gobiernos inmersos abre los canales para dar solución a la inestabilidad que a éstos aqueja.

\section{Comentarios finales}

Las explicaciones foucaultianas sobre los mecanismos de acción del poder precisan que no es un solo poder el que actúa, sino varios a la vez; asimismo, establecen que el poder no es sólo represivo, sino que también proporciona beneficios. Estos «axiomas» son indicativos de que las formas a través de las cuales se puede llevar a cabo el ejercicio biopolítico son tan vastas e ilimitadas que prácticamente no conceden margen a ningún ciudadano común para permanecer exento de alguna de estas estrategias. Sin embargo, el tema que aquí nos ocupa - la salud de los migrantes mexicanos indocumentados que viven en Estados Unidos- facilita la puesta en operación de acciones biopolíticas, esencialmente por la alta vulnerabilidad de estos ciudadanos debido a su condición de indocumentados.

Desde que los migrantes indocumentados salen de sus lugares de origen, saben que sus condiciones de vida serán distintas, ya que deberán acatar prácticamente todo tipo de mandatos (así lo hacen desde que salen de sus hogares, atendiendo las indicaciones de los «polleros»); están advertidos de que si no obedecen las disposiciones, no sólo perderán la oportunidad de cruzar la frontera, sino que sus propias vidas están en peligro. En cambio, si «son obedientes», podrán llegar a Estados Unidos y conseguirán el anhelado empleo y solventarán sus necesidades. Estos hechos, que constituyen parte de las realidades vividas por los migrantes, ponen de manifiesto las dos face- 
tas del biopoder: la parte negativa, concurrente con la obediencia y los malos tratos, y la parte positiva, cruzar la frontera y obtener el empleo deseado. Éste es el recorrido común del biopoder y esto es lo que en primera instancia sucede con los migrantes. Los mecanismos, mediante los cuales las estrategias biopolíticas de salud son incorporadas a estas poblaciones, constituyen el culmen de este ejercicio. No es gratuita la dotación de apoyos para mantenerlos saludables a través de acciones preventivas, como la aplicación de vacunas, la toma de exámenes de laboratorio, la detección oportuna de enfermedades y la promoción de medidas higiénicas, ya que el bienestar personal que proveen no es su objetivo primero sino, primordialmente, beneficiar a la propia nación estadounidense «evitando» posibles contagios provenientes de los «no ciudadanos» o indocumentados mexicanos. Estas acciones son ejemplo de las biopolíticas a que son sometidos los migrantes mexicanos. Otra estrategia biopolítica, derivada de la falta de apoyos en atención a enfermos, es el autocuidado que consiste en responsabilizar a los migrantes de su salud: es obligación de ellos cuidarse y mantenerse sanos.

En este análisis se ha podido observar cómo el discurso del poder va cambiando en correspondencia con sus propios intereses. No importan demasiado las necesidades reales de la población, en este caso, la salud de los migrantes, sino las ganancias que se obtienen de su administración y control. Los ejemplos son claros: se cambia de una estrategia biopolítica, que genera más gastos y mayores compromisos (atención preventiva y curativa), por otra de autocuidado, que rinde los mismos beneficios pero cuyo costo es menor.

Quizá esté de sobra mencionarlo, pero es necesario enfatizar lo siguiente: los intereses de Estados Unidos se sobreponen a cualquier acción del gobierno de México; el biopoder mantiene su vigencia en la medida en que, incluso por conveniencia mutua, se establece un «dominio» sobre el gobierno mexicano. 


\section{REFERENCIAS}

Agamben, Giorgio (2010), Homo Sacer. El poder soberano y la nuda vida, Valencia, Pre-Textos.

Aguilar, Cristina (2010), «Mensaje de la Comisión de Salud del Ime», Xvi Reunión Plenaria del Consejo Consultivo del Instituto, Tuxtla Gutiérrez, Chiapas, 4 de noviembre de 2010, http://www.ime.gob.mx/ime2/ images/ccime/discursos/xvi_dis_cristina_aguilar.pdf, (consulta: $20 \mathrm{de}$ enero 2012).

Becerra, Alejandro (2004), «Mecanismos de comunicación intergubernamental entre México y Estados Unidos», El cotidiano, vol. 20, núm. 127. Banco Interamericano de Desarrollo (Bid) (2010), Diez años de innovación en remesas: lecciones aprendidas y modelos para el futuro, Washington, BID.

Bossert, Thomas (2010), «La Reforma de la Salud en Estados Unidos: Acuerdos Imperfectos», Observatorio de Politicas Públicas, año 5, núm. 8.

CAstillo, Manuel Ángel (2010), «Los derechos sociales de los migrantes», en Paula Leite y Silvia E. Giorguli (coordinadoras), Reflexiones en torno a la emigración mexicana como objeto de políticas públicas, México, Conapo.

De los Ríos Lozano, Patricia (2011), «El papel de los estados en la política migratoria de Estados Unidos», Ibero, Revista de la Universidad Iberoamericana, año III, núm. 14.

EMIF (2011), «Situación de la emigración mexicana y la migración de tránsito irregular en México", La situación demográfica de México 2011, México, Conapo.

Delgado Wise, Raúl, Humberto Márquez Covarrubias y Héctor Rodríguez Ramírez (2009), «Seis tesis para desmitificar el nexo entre migración y desarrollo», Migración y desarrollo, núm. 12.

Durand, Jorge (2011), «Migración internacional y políticas públicas: inercias y desafíos», Ibero, Revista de la Universidad Iberoamericana, año III, núm.14. 
Durand, Jorge (2007), «El Programa Bracero (1942-1964). Un balance crítico», Migración y desarrollo, núm. 9.

Ferrer Lues, Marcela (2012), «Reflexiones sobre la concepción de la salud como responsabilidad individual: construyendo bioética en salud pública», en Francisco Javier León Correa (coordinador), Bioética y sociedad en Latinoamérica, Santigo, Fundación Interamericana Ciencia y Vida.

Foucault, Michel (2008), Un diálogo sobre el poder y otras conversaciones, Madrid, Filosofía Alianza. (2004), Vigilar y castigar. Nacimiento de la prisión, Buenos Aires, Siglo XXI.

(2002), Historia de la sexualidad 1. La voluntad del saber, México, Siglo XXI.

(2001), Defender la sociedad, Buenos Aires, Fondo de Cultura Económica. (1999a), «La política de salud en el siglo XviII», en Estrategias de poder, Obras esenciales, volumen II, Barcelona, Paidós.

(1999b), «Las mallas del poder», en Estética, ética y hermenéutica, Obras esenciales, volumen III, Barcelona, Paidós.

(1988), «E1 sujeto y el poder», en Hubert Dreyfus et al., Michel Foucault: más allá del estructuralismo y la hermenéutica, México, Universidad Nacional Autónoma de México.

García Canal, María Inés (2005), «Poder, violencia y palabra», Tramas, núm. 25.

GiL, Martha (2009), Poder, verdad y normalidad: genealogia del hombre moderno a través de la lectura de M. Foucault, Madrid, Cuaderno de Materiales.

Heredia Zubieta, Carlos (2011), «La migración mexicana y el debate en Estados Unidos a la sombra del Tea Party», Nueva Sociedad, núm. 233. Valencia, Roberto (2012), «Giorgio Agamben: Vida primigenia controlada por la vida política», La administración panóptica, http://administracionpanoptica.blogspot.mx/2012/05/giorgio-agamben-vida-primigenia.html (consulta: 9 mayo de 2012).

Levine, Elaine (2006), «Condiciones laborales y salariales para migrantes 
mexicanos en Estados Unidos», en Melgar Mario (coordinador), $M i-$ gración a Estados Unidos, más allá de los números, México, Universidad Nacional Autónoma de México.

Massey, Douglas, Karen Pren y Jorge Durand (2009), «Nuevos escenarios de la migración México-Estados Unidos. Las consecuencias de la guerra antiinmigrante», Papeles de Población, vol. 15, núm. 61.

Miglionico, Caino Walter (2006), Salud y trabajo. Guía para la acción sindical, Montevide, Departamento de salud laboral y medio ambiente, http// www.aebu.org.uy/doc_salud_02.pdf (consulta: 4 de diciembre de 2006). Mora, Luis (2010), «Dominación y corporalidad: técnicas de gobierno en la conquista americana», Tabula rasa, núm. 12.

Nigenda, Gustavo, José Arturo Ruiz, Rosa María Bejarano, Jacqueline A1calde, Pastor Bonilla (2009), «Análisis de las alternativas de los migrantes mexicanos en Estados Unidos de América para atender sus problemas de salud», Salud Pública de México, vol. 51, núm. 5.

Novelo, Federico (2011), «Por una política migratoria integral», Cofactor, vol. II, núm. 3.

Observatorio de la Migración (2010), «La Reforma de salud en Estados Unidos», Observatorio de la migración, http://www.observatoriodelamigracion.org/om/La_reforma_de_salud_en_Estados_Unidos.html> (consulta: 20 de enero 2012).

Ponce, Carlos (2012), «Latinos discriminados en Estados Unidos: la hipocresía de los impuestos sin beneficios», «ttp://twolatinamericas. blogspot.mx/2012/03/latinos-discriminados-en-estados-unidos. html (consulta: 2 de mayo de 2012).

ssa (2010), «Evolución de la situación migratoria», htttp://www.saludmigrante.salud.gob.mx/interior1/declaracion.html (consulta: 13 abril de 2012). (2008), Programa de Acción Especifico 2007-2012, Salud del Migrante, México, ssa.

(2002), Programa de Acción: Migrantes «Vete Sano y Regresa Sano», México, ssa.

MIGRACIÓN Y DESARROLLO, VOL. 10, NÚM. 19, SEGUNDO SEMESTRE DE 2012 \1 
TejedA, José (2011), «Biopolítica, control y dominación», Espiral, vol. xviıI, núm. 52.

Universidad de California y ssa, (2007), Migración, salud y trabajo: datos frente a los mitos, México, ssA, uc. 